\title{
Avaliação do modelo de pressão e temperatura global da Universidade de Viena com dados de sensores meteorológicos no Brasil
}

\author{
Evaluation of the global pressure and temperature model of University of Vienna with meteorological sensors data in \\ Brazil
}

\begin{abstract}
Wagner Carrupt Machado 1,2
Edvaldo Simões da Fonseca Junior ${ }^{2}$
${ }^{1}$ Gerência de Geodésia e Cartografia da Unidade Estadual do Instituto Brasileiro de Geografia e Estatística em Santa Catarina - IBGE. Rua Tenente Silveira, 94, Cep 88010-300. Florianópolis, SC, Brasil.
${ }^{2}$ Departamento de Engenharia de Transportes - Laboratório de Topografia e Geodésia, Universidade de São Paulo USP. Avenida Almeida Prado, travessa 2, n 83, Cidade Universitária. Cep 05508-070. São Paulo, SP, Brasil.
\end{abstract}

wagner@carrupt.com.br, edvaldo@usp.br

\begin{abstract}
RESUMO - Os sinais GNSS sofrem refração ao se propagarem pela atmosfera neutra, introduzindo o atraso troposférico, que pode chegar a cerca de $25 \mathrm{~m}$, nas medidas de distância entre os satélite e o receptor. Devido às características da atmosfera neutra, é conveniente dividir o atraso troposférico em duas componentes: a hidrostática e a úmida. Modelos que requerem informações de pressão, temperatura e umidade relativa do ar, tais como o desenvolvido por Hopfield, podem ser utilizados para minimizar os efeitos desta camada da atmosfera nos sinais GNSS, sobretudo da componente hidrostática. As informações meteorológicas podem ser obtidas a partir de sensores meteorológicos, modelos de previsão numérica do tempo (PNT), redução de dados de uma atmosfera padrão ou de modelos empíricos, como o modelo de pressão e temperatura global (Global Pressure and Temperature - GPT) que foi desenvolvido na Universidade de Viena, Áustria. Dentre as opções citadas, a última se destaca devido à facilidade de uso. Esta pesquisa apresenta uma comparação entre as grandezas produzidas pelo modelo GPT com dados observados em dez estações meteorológicas automáticas (EMA) do Instituto Nacional de Meteorologia (INMET), localizadas em diferentes regiões do Brasil. Os experimentos foram conduzidos com dados do ano de 2010. Análises acerca da diferença de pressão e da diferença de temperatura mostram que o modelo GPT calcula as grandezas meteorológicas com acurácia de 4,3 hPa e $4,5^{\circ} \mathrm{C}$, respectivamente.
\end{abstract}

Palavras-chave: GPT, dados meteorológicos, modelagem da atmosfera neutra.

ABSTRACT - GNSS signals are refracted when propagating through the neutral atmosphere, introducing the tropospheric delay, that can reach about $25 \mathrm{~m}$, in the satellite-receiver distances. Due to the properties of the atmosphere, it is interesting to divide the tropospheric delay into 2 components: hydrostatic and wet. Models which require information on pressure, temperature and relative humidity of the air, as the one developed by Hopfield, can be used to mitigate the neutral atmosphere effects on GNSS signals, mainly of hydrostatic component. The meteorological data can be obtained from meteorological sensors, numerical weather predictions models, standard atmosphere reduction or empirical models, as the global pressure and temperature (GPT) model developed at University of Viena. Among the options cited, the empirical model can be highlighted by the facility of implementation. This research presents a comparison between the meteorological data computed by the GPT model and data from ten automatic meteorological stations in different regions of Brazil. Data from 2010 were used in the experiment. Analysis on pressure differences and temperature differences show that GPT computes such meteorological data with, respectively, 4,3 hPa and $4,5^{\circ} \mathrm{C}$ of accuracy..

Keywords: GPT, meteorological data, neutral atmospheric modeling.

\section{INTRODUÇÃO}

O posicionamento com os sistemas de navegação global por satélite (GNSS) é obtido em função da velocidade e do tempo de propagação dos sinais emitidos pelos satélites. Se tais sinais se propagassem no vácuo, a velocidade de propagação seria igual à velocidade da luz neste meio. No entanto, durante a propagação entre a antena dos satélites e a antena do receptor, os sinais GNSS interagem com partículas carregadas, bem como com moléculas e átomos neutros, presentes na atmosfera. Isto causa distúrbios na potência e a refração do sinal que provocam variação na velocidade e direção de propagação (LANGLEY, 1998, SEEBER, 2003). 
A camada da atmosfera neutra mais próxima da superfície terrestre, até aproximadamente $12 \mathrm{~km}$, é caracteriza pela presença de vapor d'água. Este último é responsável pelo atraso troposférico úmido dos sinais GNSS $\left(\operatorname{Trop}_{u}\right)$. Além da presença do vapor d'água, a atmosfera neutra é constituída de uma mistura de gases secos, provocando o atraso troposférico hidrostático $\left(\operatorname{Trop}_{h}\right)$. Destaca-se que a refração provocada pela atmosfera neutra pode resultar em erros na medida da distância satélite-receptor que variam de $2 \mathrm{~m}$ a $25 \mathrm{~m}$, dependendo do ângulo de elevação (SPILKER Jr, 1996).

Portanto, o atraso troposférico é resultante da combinação entre o atraso nas duas componentes, sendo escrito como (PETIT; LUZUM, 2010):

$$
\begin{aligned}
\text { Trop }= & \operatorname{Trop}_{h}^{Z} m_{h}(\text { ele })+\operatorname{Trop}_{u}^{Z} m_{u}(\text { ele })+ \\
& +m_{g}(\text { ele })\left(G_{N} \cos (a z)+G_{E} \operatorname{sen}(a z)\right)
\end{aligned}
$$

na qual $\operatorname{Trop}_{h}^{Z}$ e $\operatorname{Trop}_{u}^{Z}$ são o atraso troposférico hidrostático e úmido na direção zenital, respectivamente, $m_{h}, m_{u}$ e $m_{g}$ representam, respectivamente, as funções de mapeamento das componentes hidrostática e úmida e do gradiente do atraso horizontal, $G_{N}$ e $G_{E}$ são as componentes do gradiente e ele e az são a elevação e o azimute do satélite.

A componente hidrostática é responsável por aproximadamente $90 \%$ dos efeitos causados pela atmosfera neutra. Esta componente possui comportamento uniforme, sendo modelada com boa acurácia através de modelos que requerem medidas de pressão e temperatura, ou apenas de pressão. Tais medidas devem ser reduzidas à altitude da antena do receptor. Sapucci e Monico (2001) afirmam que se a medida de pressão for realizada com precisão de $0,5 \mathrm{hPa}$, o atraso $\operatorname{Trop}_{h}^{Z}$ pode ser determinado com incerteza de $1 \mathrm{~mm}$. Por outro lado, a componente úmida varia bastante no tempo e no espaço e seu comportamento é de difícil modelagem (SPILKER Jr, 1996).

$\mathrm{Na}$ utilização dos modelos citados, as grandezas meteorológicas podem ser obtidas com sensores calibrados e com precisão compatível. Isto implica na necessidade do usuário adquirir tais sensores, bem como realizar a medida durante o rastreio. Uma alternativa seria utilizar dados de um modelo de previsão numérica do tempo (PNT), como os utilizados pelo Centro de Previsão de Tempo e Estudos Climáticos do Instituto Nacional de Pesquisas Espaciais (CPTEC/INPE) (INPE, 2013). Uma aproximação mais grosseira seria utilizar valores obtidos da redução de uma atmosfera padrão no elipsóide para a altitude da antena do receptor. Esta estratégia é utilizada em programas de análise de dados GNSS, tais como o Bernese e o GAMIT, para calcular o valor inicial do $\operatorname{Trop}^{Z}$, que é introduzido como incógnita adicional no ajustamento das observações (HUGENTOBLER et al. 2006; KING; BOCK, 2006).

O modelo de pressão e temperatura global (GPT Global Pressure and temperature), desenvolvido na universidade de Viena, é uma alternativa a se utilizar valores de uma atmosfera padrão. Uma comparação entre a pressão oriunda desta última estratégia com o modelo GPT, em escala global, mostrou que a utilização da atmosfera padrão tem seu desempenho degradado em parte do hemisfério sul (BOEHM; HEINKELMANN; SCHUH, 2007).

Esta pesquisa apresenta uma avaliação do modelo GPT no Brasil, onde as medidas de pressão e temperatura calculadas com tal modelo foram comparadas com medidas de sensores de dez estações meteorológicas automáticas (EMA) da rede do Instituto Nacional de Meteorologia (INMET, 2011) distribuídas em regiões distintas do Brasil durante o ano de 2010.

\section{MODELAGEM DA ATMOSFERA NEUTRA E O MODELO DE PRESSÃO E TEMPERATURA GLOBAL}

Os efeitos provocados pela passagem dos sinais GNSS através da atmosfera neutra podem ser adequadamente modelados em função da refratividade do ar. No entanto, devido à dificuldade de se medir tal grandeza ao longo da trajetória de propagação do sinal, ou até mesmo ao longo da direção zenital, foram desenvolvidos modelos visando o cálculo do atraso troposférico zenital $\left(\operatorname{Trop}^{\mathrm{Z}}\right)$. Dentre os modelos desenvolvidos se destaca o de Hopfield (HOPFIELD, 1969), onde dados de pressão, temperatura e umidade relativa do ar reduzidas à altitude da antena do receptor são necessários. Este modelo é dado por (SEEBER, 2003):

$$
\begin{aligned}
& \operatorname{Trop}_{h}^{Z}=155,2 \times 10^{-7} \frac{P_{s}}{T_{s}} H_{h} \\
& \operatorname{Trop}_{u}^{Z}=155,2 \times 10^{-7} \frac{4810 e_{s}}{T_{s}^{2}} H_{u}
\end{aligned}
$$

onde $P_{s}$ e $T_{s}$ representam a pressão e temperatura atmosférica na altitude da antena, respectivamente, $H_{h}$ e $H_{u}$ representam, respectivamente, a altura das camadas atmosféricas das componentes hidrostáticas e úmida em unidade métrica, dadas por:

$$
\begin{aligned}
& H_{h}=40136+148,72\left(T_{s}-273,16\right) \\
& H_{u}=11000
\end{aligned}
$$

Uma aproximação para o termo $e_{s}$ da Eq. (3) pode ser obtida através da seguinte equação (LEICK, 2004):

$$
e_{s} \frac{U R \times e^{-37,2465+0,213166 T-0,000256908 T^{2}}}{100}
$$

onde $U R$ é a umidade relativa (\%).

Devido, principalmente, à dificuldade de se modelar a componente úmida, programas de análise de dados 
GNSS, tais como o Bernese e o GAMIT, utilizam os modelos empíricos para calcular o valor inicial do $\operatorname{Trop}^{Z}$, que é estimado no ajustamento das observações juntamente com os demais parâmetros (HUGENTOBLER et al. 2006; KING; BOCK, 2006).

\subsection{Obtenção das grandezas meteorológicas}

As grandezas meteorológicas, necessárias para a modelagem do atraso troposférico a partir do modelo de Hopfield, podem ser obtidas a partir de medições provenientes de sensores meteorológicos, da redução de grandezas de uma atmosfera padrão, de modelos de previsão numérica do tempo (PNT) ou de modelos empíricos. Dentre os métodos citados, a utilização do modelo empírico se destaca devido à facilidade de uso, se comparado com o emprego de sensores meteorológicos ou de modelos de PNT. Além disso, esta estratégia apresenta melhor desempenho para o hemisfério sul do que se utilizar uma atmosfera padrão, conforme pode ser visto na Tab. 1 e na Fig. 1 (SAPUCCI, 2001; SAPUCCI; MACHADO; MONICO, 2006; BOEHM; HEINKELMANN; SCHUH, 2007).

O GPT foi desenvolvido na Universidade de Viena. $\mathrm{Na}$ determinação deste modelo foram utilizados dados de médias mensais de perfis de pressão e temperatura, bem como de altitudes ortométricas, de uma grade global de $15^{\circ}$ x $15^{\circ}$ de três anos (setembro de 1999 a agosto de 2002) do European Centre for Medium-Range Weather Forecasts (ECMWF). Tais dados foram reduzidos ao nível médio dos mares e tiveram suas médias e amplitudes anuais calculadas com deslocamento de fase fixada em 28 de janeiro. As quatro grades resultantes foram expandidas em uma série de funções harmônicas esféricas de grau e ordem nove. Os parâmetros de entrada do GPT são as coordenadas geodésicas tridimensionais da estação e a data juliana modificada (BOEHM; HEINKELMANN; SCHUH, 2007).

Tabela 1 - Médias e desvios-padrão da diferença entre a pressão e a temperatura medidas por estações meteorológicas e obtidas com o modelo GPT e utilização de atmosfera padrão durante 2005 (Adaptado de: BOEHM; HEINKELMANN; SCHUH, 2007).

\begin{tabular}{lccccc}
\hline \multicolumn{1}{c}{ Estação } & $\begin{array}{c}\text { Latitude } \\
\left({ }^{\circ}\right)\end{array}$ & $\begin{array}{c}\text { Pressão } \\
(\mathbf{h P a}) \\
\text { Met. }- \text { GPT }\end{array}$ & $\begin{array}{c}\text { Pressão (hPa) } \\
\text { Met - Berg }\end{array}$ & $\begin{array}{c}\text { Temperaura }\left({ }^{\circ} \mathbf{C}\right) \\
\text { Met }- \text { GPT }\end{array}$ & $\begin{array}{c}\text { Temperaura }\left({ }^{\circ} \mathbf{C}\right) \\
\text { Met- Eq2 }\end{array}$ \\
\hline Algonquin Park & 46 & $5,7 \pm 7,4$ & $2,9 \pm 7,3$ & $0,5 \pm 7,2$ & $-10,2 \pm 13,6$ \\
Hartebeenthoek & -26 & $2,8 \pm 3,4$ & $11,0 \pm 3,7$ & $0,3 \pm 6,2$ & $8,8 \pm 6,4$ \\
Kokee Park & 22 & $4,4 \pm 2,3$ & $8,6 \pm 2,3$ & $-0,3 \pm 2,6$ & $6,5 \pm 2,8$ \\
Ny-Alesund & 78 & $0,7 \pm 12,9$ & $-2,9 \pm 12,7$ & $0,0 \pm 5,4$ & $-20,3 \pm 5,9$ \\
Westforf & 49 & $1,2 \pm 7,4$ & $-0,5 \pm 7,4$ & $2,3 \pm 5,8$ & $-4,9 \pm 10,9$ \\
Wettzell & 42 & $2,7 \pm 7,1$ & $10,8 \pm 7,0$ & $0,3 \pm 4,8$ & $-4,9 \pm 8,4$ \\
\hline
\end{tabular}

Boehm, Heinkelmann e Schuh (2007) realizaram uma comparação da pressão e temperatura calculada com o modelo GPT com a utilização de dados de uma atmosfera padrão. Tal avaliação foi realizada considerando medidas meteorológicas coletadas dois dias por semana durante o ano de 2005. Os resultados do experimento são apresentados na Tab. 1.

Observa-se que a tendência da pressão obtida com o GPT é menor do que a da atmosfera padrão em 4 das 6 estações, incluindo a única estação localizada no hemisfério sul. Verifica-se, ainda, que a tendência da temperatura das seis estações é menor quando se utilizou o modelo GPT.

A Fig.1 mostra a diferença de pressão entre o modelo GPT e a pressão obtida da redução de uma atmosfera padrão em escala global.

A comparação da pressão produzida pelo GPT com a pressão calculada a partir de uma atmosfera padrão revela que esta última possui uma tendência em parte do hemisfério sul, atingindo o valor de aproximadamente 47 hPa na latitude $-80^{\circ}$.

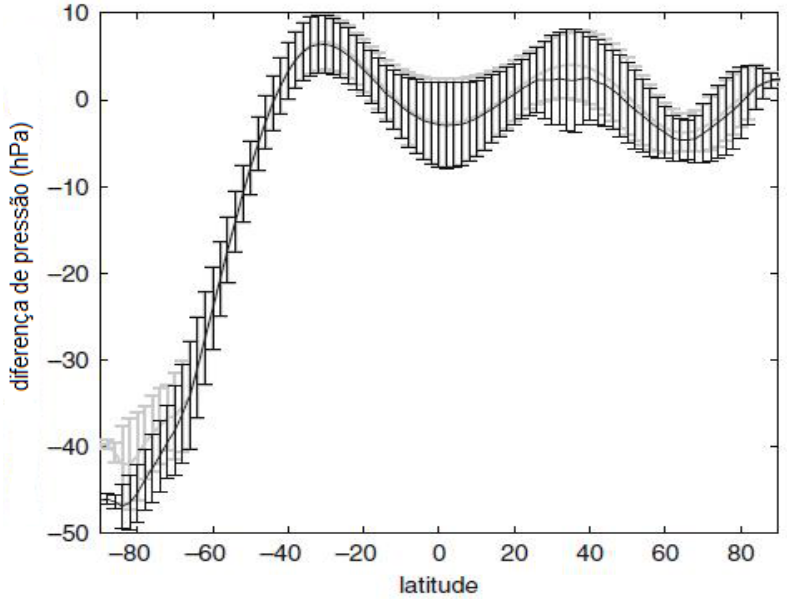

Figura 1 - Diferença de pressão entre o modelo GPT e atmosfera padrão.

Fonte: Adaptado de Boehm, Heinkelmann e Schuh (2007).

\section{EXPERIMENTOS}

\subsection{Conjunto de dados}

Dados de pressão e temperatura do modelo GPT foram comparados com dados observados em dez EMAs pertencentes à rede do INMET durante o ano de 2010. Tais estações estão localizadas nos municípios de 
Manaus/AM, Belém/PA, Macapá/AP, Belo Horizonte/MG, Vitória/ES, Campo Grande/MS, Ilha Solteira/SP, Barra do Turvo/SP e Laguna/SC. A Fig. 2 mostra a distribuição das EMAs.

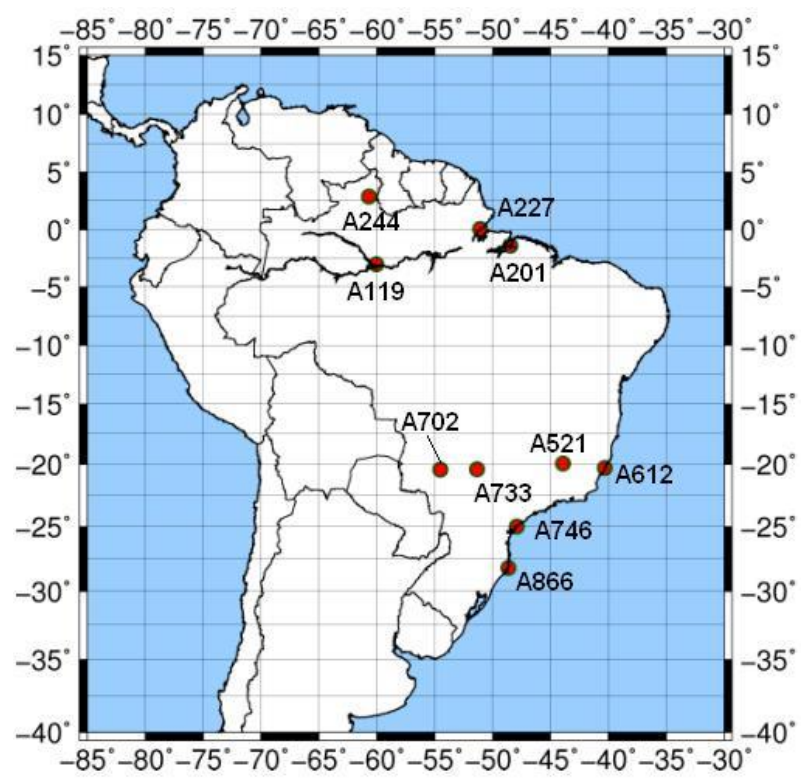

Figura 2 - Distribuição das EMAs.

Conforme pode ser observado na Fig. 2, as EMAs estão distribuídas em regiões distintas do território brasileiro, compreendendo regiões próximas ao mar, no interior do país e sob influência do Rio Amazonas.

Além da localização geográfica, a altitude exerce influência sobre as condições atmosféricas. A Fig. 3 traz um gráfico com a altitude aproximada das EMAs.

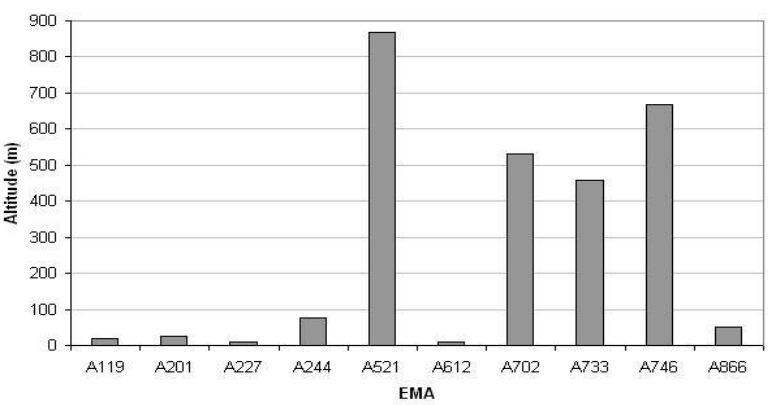

Figura 3 - Altitude das EMAs.

Como pode ser observado, além das EMAs estarem distribuídas em locais geograficamente distintos, elas possuem altitude que varia do nível do mar (A227 e A612) até a, aproximadamente, 860 m (A521).

\subsection{Resultados}

Assumindo as medidas de pressão e temperatura observadas nas EMAs como referência, foram calculadas diferenças com relação às grandezas calculadas com o modelo GPT. A Fig. 4 traz a série temporal da diferença de pressão na estação A 612, localizada em Vitória/ES.

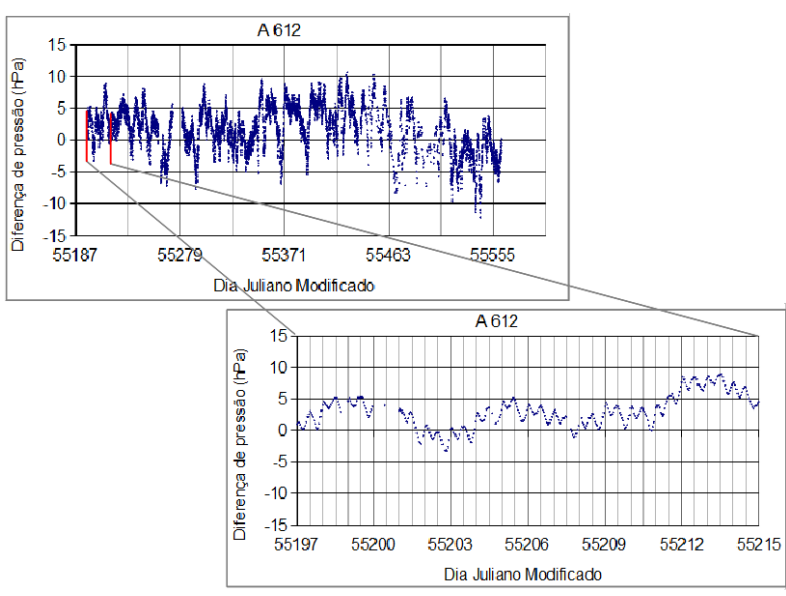

Figura 4 - Série temporal da diferença de pressão (A 612).

Verifica-se que, fora o período de primavera, a diferença de pressão apresenta um comportamento cíclico em torno de um valor médio. $\mathrm{Na}$ parte da figura que destaca um trecho de 18 dias da série temporal, podem ser observados ciclos de aproximadamente 6 dias e ciclos mais curtos e de menor magnitude que duram cerca de 24 horas. Salienta-se que resultados similares foram obtidos na maioria das demais estações.

A tabela 2 apresenta as diferenças máxima, média, mínima, diferença média quadrática (RMS) e desviopadrão para a pressão obtidos nas dez estações.

Tabela 2 - Estatísticas da diferença de pressão.

\begin{tabular}{llllll}
\hline Estação & $\begin{array}{c}\text { Máximo } \\
(\mathbf{h P a})\end{array}$ & $\begin{array}{c}\text { Média } \\
(\mathbf{h P a})\end{array}$ & $\begin{array}{c}\text { Mínimo } \\
(\mathbf{h P a})\end{array}$ & $\begin{array}{c}\text { RMS } \\
\mathbf{( h P a})\end{array}$ & $\begin{array}{c}\text { Desvio- } \\
\text { padrão } \\
\text { (hPa) }\end{array}$ \\
\hline A119 & 4,5 & $-4,1$ & $-11,6$ & 4,6 & 2,1 \\
$\mathrm{~A} 201$ & 9,9 & 2,7 & $-4,1$ & 3,2 & 1,7 \\
$\mathrm{~A} 227$ & 9,9 & 2,5 & $-4,1$ & 3,0 & 1,7 \\
A244 & 11,9 & 3,5 & $-3,8$ & 4,0 & 1,9 \\
A521 & 12,3 & 3,7 & $-9,4$ & 4,9 & 3,2 \\
A612 & 10,7 & 1,8 & $-12,1$ & 4,0 & 3,5 \\
A702 & 8,1 & $-2,8$ & $-17,5$ & 4,4 & 3,4 \\
A733 & 7,0 & $-2,1$ & $-13,0$ & 3,9 & 3,3 \\
A746 & 15,3 & 4,1 & $-10,0$ & 5,8 & 4,0 \\
A866 & 12,4 & 0.9 & $-12,9$ & 4,5 & 4,4 \\
\hline
\end{tabular}

Observa-se que, em média, o modelo GPT subestimou o valor da pressão em 7 das 10 estações, resultando em uma tendência de $1,1 \mathrm{hPa}$ quando se considera todas as estações. Além disso, verifica-se que a acurácia da determinação da pressão pelo GPT, dada pela RMS, varia de $3,0 \mathrm{hPa}$ a 5,8 hPa e é da ordem de 4,3 hPa ao se considerar as diferenças em todas as estações. Comparando a RMS com o desvio-padrão das diferenças de pressão constata-se a presença de erros sistemáticos que variam de $0,1 \mathrm{hPa}$ a 2,5 hPa, nas estações A866 e A119, respectivamente. Ao se analisar as diferenças espacialmente, constata-se que o desempenho da determinação de pressão do GPT é similar em todas as estações.

A série temporal da diferença de temperatura na estação A 612 é apresentada na Fig. 5. 


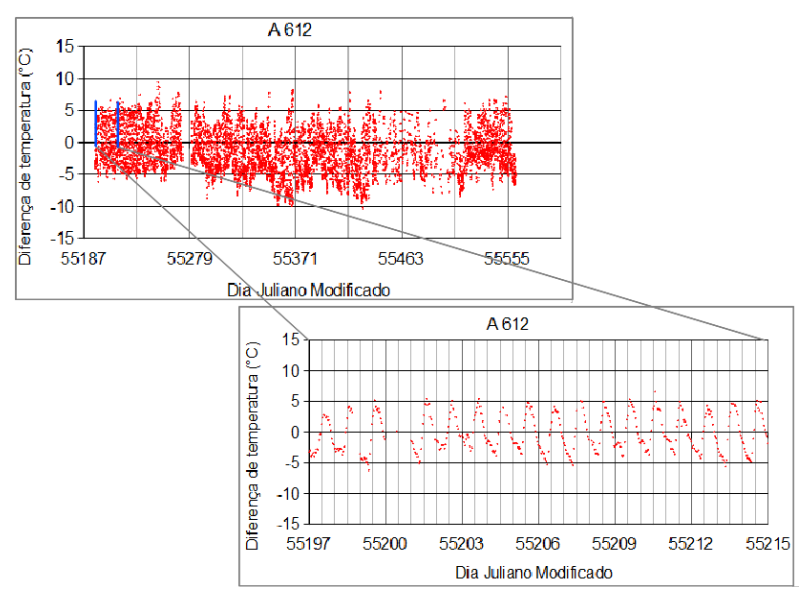

Figura 5 - Série temporal da diferença de pressão (A612).

Observa-se que a diferença de temperatura também apresenta variação cíclica em torno de um valor médio durante todo o período da análise. No destaque podem ser verificados ciclos de aproximadamente 24 horas, os quais estão presentes em toda a série. Salienta-se que resultados similares foram obtidos nas demais estações.

A Tab. 3 apresenta as diferenças máxima, média, mínima, diferença média quadrática (RMS) e desviopadrão para a temperatura obtidos nas dez estações consideradas.

Tabela 3 - Estatísticas da diferença de temperatura

\begin{tabular}{llllll}
\hline Estação & $\begin{array}{c}\text { Máximo } \\
\left({ }^{\circ} \mathbf{C}\right)\end{array}$ & $\begin{array}{c}\text { Média } \\
\left({ }^{\circ} \mathbf{C}\right)\end{array}$ & $\begin{array}{c}\text { Mínimo } \\
\left({ }^{\circ} \mathbf{C}\right)\end{array}$ & $\begin{array}{l}\text { RMS } \\
\left({ }^{\circ} \mathbf{C}\right)\end{array}$ & $\begin{array}{c}\text { Desvio- } \\
\text { padrão } \\
\left({ }^{\circ} \mathbf{C}\right)\end{array}$ \\
\hline A119 & 7,7 & $-1,5$ & $-9,4$ & 3,1 & 2,8 \\
A201 & 7,8 & 0,1 & $-5,1$ & 2,8 & 2,8 \\
A227 & 6,3 & 1,1 & $-4,7$ & 2,2 & 1,9 \\
A244 & 8,8 & $-0,9$ & $-6,4$ & 3,0 & 2,9 \\
A521 & 7,4 & $-4,4$ & $-16,9$ & 5,7 & 3,7 \\
A612 & 9,4 & $-1,5$ & $-10,2$ & 3,7 & 3,4 \\
A702 & 12,3 & $-3,2$ & $-19,1$ & 6,1 & 5,2 \\
A733 & 13,5 & $-1,6$ & $-16,8$ & 5,2 & 4,9 \\
A746 & 8,8 & $-6,1$ & $-19,6$ & 7,3 & 3,9 \\
A866 & 7,3 & $-2,0$ & $-8,6$ & 2,8 & 2,0 \\
\hline
\end{tabular}

A Tab. 3 mostra que, na média, o modelo GPT superestimou o valor da temperatura em 8 das 10 estações consideradas e que a acurácia desta grandeza variou de $2,2{ }^{\circ} \mathrm{C}$ a $7,3{ }^{\circ} \mathrm{C}$, sendo de $4,5{ }^{\circ} \mathrm{C}$ quando se considera as diferenças em todas as estações. Destaca-se, também, que o efeito sistemático é menor que $1^{\circ} \mathrm{C}$ em 8 das 10 estações, sendo praticamente nulo na estação A201. Por outro lado, nas estações A746 e A521 tal efeito é de 3,4 ${ }^{\circ} \mathrm{C}$ e $2{ }^{\circ} \mathrm{C}$, respectivamente. Observa-se que os resultados com pior acurácia foram obtidos nas estações com altitude superior a $400 \mathrm{~m}$.

\section{CONCLUSÕES E CONSIDERAÇÕES FINAIS}

Medidas de pressão e da temperatura obtidas com o modelo GPT foram comparadas com observações meteorológicas obtidas de dez EMAs distribuídas em regiões distintas do Brasil durante 2010. Os resultados mostram que o GPT determinou tais grandezas com acurácia de $4,3 \mathrm{hPa}$ e $4,5^{\circ} \mathrm{C}$, respectivamente.

Além disso, observa-se que as diferenças são centradas em torno de um valor médio durante o período do experimento. Enquanto a diferença de pressão apresenta um ciclo de aproximadamente 6 dias e um de menor magnitude e duração de 24 horas a diferença de pressão apresenta ciclos com período de 24 horas.

Pretende-se incluir as grandezas meteorológicas obtidas da redução de uma atmosfera padrão em análises futuras, bem como o impacto das diferenças sobre o valor do atraso troposférico hidrostático.

\section{AGRADECIMENTOS}

O primeiro autor gostaria de agradecer ao IBGE pela concessão de afastamento para a realização de parte desta pesquisa. Agradecemos, também, o Dr. Luiz Fernando Sapucci e o Dérek Schubert, do CPTEC/INPE, pela disponibilização dos dados meteorológicos.

\section{REFERÊNCIAS}

BOEHM, J.; HEINKELMANN, R.; SCHUH, H. Short note: a global model of pressure and temperature for geodetic applications. Journal of Geodesy, v. 81, n. 10, p. 679-683, Oct., 2007.

HOPFIELD, H. S. Two-quadratic tropospheric refractivity profile for correcting satellite data. Journal of Geophysical Research, v. 74, n. 18,p. 4487- 4499, Aug., 1969.

HUGENTOBLER, U.; DACH, R.; FRIDEZ, P.; MEINDL, M. Bernese GPS Software Version 5.0. Astronomical Institute, University of Berne, 2007.

INMET Rede de estações meteorológicas automáticas do INMET. Nota $\mathrm{n}^{\circ}$ 001/2011/SEGER/LAIME/CSC/INMET, 2011.

INPE Previsão numérica: modelos regionais e globais, 2013. Disponível em: <http://previsaonumerica.cptec.inpe.br/golMapWeb/D adosPages?id=Eta15>. Acesso em 09 de jan. 2013.

KING, R. W.; BOCK, Y. Documentation for the GAMIT GPS processing software Release 10.2. MIT, Cambridge Lemoine FG, Kenyon SC, Factor JK, Trimmer RG, Pavlis, 2006.

LANGLEY, R. B. Propagation of the GPS signals. In: TEUNISSEN, P. J. G.; KLEUSBERG, A. GPS for Geodesy. 2.ed.: Springer Verlag, 1998. pp. 111-149.

LEICK, A. GPS satellite surveying. $3^{\mathrm{a}}$ ed. New Jersey: John Wiley \& Sons, 2004. 435 p.

PETIT, G.; LUZUM, B. IERS Conventions (2010): IERS Technical Note $\mathrm{N}^{\circ}$ 36, Frankfurt am Main: Verlag des Bundesamts für Kartographie und Geodäsie, 2010. 179 p.

SAPUCCI, L. F. Estimativa do vapor d'água atmosférico e avaliação da modelagem do atraso zenital troposférico utilizando GPS. 2001. 167 p. Dissertação (Mestrado) - Faculdade de Ciências e Tecnologia, Universidade Estadual Paulista, Presidente Prudente, 2001. 
SAPUCCI, L. F.; MACHADO, L. A. T.; MONICO, J. F. G. Previsões do atraso zenital troposférico para a América do Sul: variabilidade sazonal e avaliação da qualidade. Revista Brasileira de Cartografia, n. 58/03, dez., 2006.

SEEBER, G. Satellite geodesy. $2^{\mathrm{a}}$ ed. Berlim: de Gruyter, 2003. p. 589.

SPILKER Jr., J. J. Tropospheric effects on GPS. In Parkinson B. W. e Spilker Jr. J .J. Global Positioning System: Theory and Applications, Volume 1, Cambridge American Institute of Aeronautics and Astronautics, v. 163, p. 517 - 546, 1996. 